\title{
Dialogische Textkompetenz - Routinisiertes Schreiben in studentischer Online-Teamarbeit
}

\author{
Lindemann, Katrin ; Ruoss, Emanuel ; Weinzinger, Caroline
}

\begin{abstract}
Current concepts in writing research primarily focus on monological texts, by which we mean texts that do not demand a reply. But nowadays, dialogical writing - the exchange of messages via e.g. email, text message or internet forum - is increasingly prevalent in private, educational and professional life. We therefore argue that concepts of writing research should also be made applicable to dialogical writing. Based on empirical data from two university e-learning classes, we show how students use communicative routines in order to manage a specific (writing) task: During their group work students face the challenge of initiating new steps and mobilising other group members to proceed with the project. Our study shows that texts accomplishing this task usually follow a three-part structure: They give reasons why writing to the group becomes necessary ("account"), they request the start or continuation of working ("projection") and they present a personal contribution to the task ("achievement/input"). In cooperative online work, appropriate dialogical writing is a crucial skill. This "dialogical text competence", as we call it, cannot be taken for granted; indeed it must be taught and practiced as it differs from competences necessary in face-to-face interaction or for writing texts in non-dialogical contexts. We therefore close our paper with a discussion of our results under the aspect of learning and facilitating dialogical text competence in contexts that offer practical experience.
\end{abstract}

DOI: https://doi.org/10.1515/zfal-2016-0021

Posted at the Zurich Open Repository and Archive, University of Zurich ZORA URL: https://doi.org/10.5167/uzh-126267

Journal Article

Published Version

Originally published at:

Lindemann, Katrin; Ruoss, Emanuel; Weinzinger, Caroline (2016). Dialogische Textkompetenz - Routinisiertes Schreiben in studentischer Online-Teamarbeit. ZFAL, 65(1):159-182.

DOI: https://doi.org/10.1515/zfal-2016-0021 


\section{Katrin Lindemann*, Emanuel Ruoss und Caroline Weinzinger Dialogische Textkompetenz - Routinisiertes Schreiben in studentischer Online-Teamarbeit}

\section{Dialogical Text Competence: Writing Routines in Online Teams}

DOI 10.1515/zfal-2016-0021

Abstract: Current concepts in writing research primarily focus on monological texts, by which we mean texts that do not demand a reply. But nowadays, dialogical writing - the exchange of messages via e.g. e-mail, text message or internet forum is increasingly prevalent in private, educational and professional life. We therefore argue that concepts of writing research should also be made applicable to dialogical writing. Based on empirical data from two university e-learning classes, we show how students use communicative routines in order to manage a specific (writing) task: During their group work students face the challenge of initiating new steps and mobilising other group members to proceed with the project. Our study shows that texts accomplishing this task usually follow a three-part structure: They give reasons why writing to the group becomes necessary ("account"), they request the start or continuation of working ("projection") and they present a personal contribution to the task ("achievement/input"). In cooperative online work, appropriate dialogical writing is a crucial skill. This "dialogical text competence", as we call it, cannot be taken for granted; indeed it must be taught and practiced as it differs from competences necessary in face-to-face interaction or for writing texts in non-dialogical contexts. We therefore close our paper with a discussion of our results under the aspect of learning and facilitating dialogical text competence in contexts that offer practical experience.

Keywords: cooperative work, dialogical writing, e-learning, internet forum, text competence, writing routines

\footnotetext{
*Korrespondenzautor: Katrin Lindemann, Universität Zürich, Deutsches Seminar, Schönberggasse 9, CH-8001 Zürich, E-mail: katrin.lindemann@ds.uzh.ch

Emanuel Ruoss, Universität Zürich, Deutsches Seminar, Schönberggasse 9, CH-8001 Zürich, E-mail: emanuel.ruoss@ds.uzh.ch
}

Caroline Weinzinger, Universität Zürich, Deutsches Seminar, Schönberggasse 9, CH-8001 Zürich, E-mail: caroline.weinzinger@ds.uzh.ch 


\section{Einleitung}

Kommunikative Prozesse im Berufsleben, aber auch in Ausbildungskontexten, sind heute vom intensiven Gebrauch digitaler Medien geprägt. Die Internationalisierung und die technischen Möglichkeiten der Telearbeit führen dazu, dass Teams z. B. in größeren Unternehmen teilweise sogar vorrangig virtuell kommunizieren. Schriftlichen Kommunikationsformen, mit denen zeitund ortsunabhängig, d. h. asynchron, korrespondiert wird, kommt dabei besondere Bedeutung zu.

Die Entwicklung hin zur kooperativen Teamarbeit mittels elektronischer Schriftlichkeit stellt neue kommunikative Anforderungen an die Beteiligten. Diese Anforderungen sind besonders hoch, wenn es darum geht, unter den Bedingungen asynchroner Kommunikation mit schriftsprachlichen Mitteln gemeinsame Arbeits- oder Lernprozesse erfolgreich zu koordinieren und $\mathrm{zu}$ vollziehen. Gefragt ist hier nicht mehr nur das Wissen um die formale Gestaltung von Textsorten in spezifischen Kommunikationsformen, wie z. B. den angemessenen Sprachgebrauch in geschäftlichen E-Mails. Vielmehr gewinnt die dialogische Textkompetenz der Beteiligten zunehmend an Bedeutung. Damit meinen wir die Fähigkeit, Texte nicht nur rezipientenorientiert $\mathrm{zu}$ verfassen, ${ }^{1}$ sondern auch so, dass Anschlussmöglichkeiten für Antworttexte - und damit ein strukturelles Potential für eine dialogische Sequenz - eröffnet werden: Dialogisch orientierte Texte sind so strukturiert, formuliert und platziert, dass sie im Sinne der Rezipientenorientierung auf die kommunikativen Erwartungen und Voraussetzungen der übrigen Beteiligten sowie die Gegebenheiten der Kommunikationssituation zugeschnitten sind und darüber hinaus ein reibungsloses und aufgaben- bzw. zielorientiertes Hin-und-Her von Texten ermöglichen. Text-Praktiken, die dialogischer Natur sind, werden zwar in Textkompetenzmodellen gelegentlich genannt (vgl. z. B. Kleinberger Günther/Wagner 2010), ihre empirische Erforschung stellt jedoch nach wie vor ein Desiderat dar.

Unter Bezugnahme auf konversationsanalytische und textlinguistische Konzepte stellen wir in diesem Beitrag anhand natürlicher Daten ${ }^{2}$ aus einem universitären E-Learning-Kurs zwei kommunikative Verfahren vor, die von

1 Vgl. grundlegend zu recipient design Sacks/Schegloff 1979; Schegloff 1972, 1996.

2 Im Gegensatz zu vielen anderen Forschungsrichtungen plädiert die Gesprächs / Konversationsanalyse für die Verwendung sog. ,natürlicher` Gespräche, die auch ohne die Datenerhebung stattgefunden hätten (vgl. z. B. Psathas 1990: 3 4). 
Studierenden eingesetzt werden, um Arbeitsprozesse in einer Gruppe mit grundsätzlich gleichberechtigten Beteiligten zu initiieren. ${ }^{3}$

\section{Dialogische Textkompetenz und routinisiertes Schreiben}

Unter dialogischer Schriftlichkeit verstehen wir Formen von „wechselseitig mittelbarem kommunikativen Handeln“ (Luckmann 1988: 54, zit. bei Günthner 2011: 7) in und durch Schrift. Im Kontext der zunehmenden Bedeutung solcher Formen dialogischer Schriftlichkeit, bei der im Gegensatz zu monologischen Texten eine schriftliche Reaktion des Gegenübers relevant wird, plädieren wir dafür, gängige Konzepte der Schreibforschung auf ihre Bedeutung für schriftdialogische Kontexte zu überprüfen.

Die Erkenntnisse der Schreibforschung zum Konzept der Textkompetenz sind umfassend und divers. Eine erste Annäherung formuliert PortmannTselikas wie folgt:

Textkompetenz ermöglicht es, Texte selbständig zu lesen, das Gelesene mit den eigenen Kenntnissen in Beziehung zu setzen und die dabei gewonnenen Informationen und Erkenntnisse für das weitere Denken, Sprechen und Handeln zu nutzen. Textkompetenz schließt die Fähigkeit ein, Texte für andere herzustellen und damit Gedanken, Wertungen und Absichten verständlich und adäquat mitzuteilen. (Portmann Tselikas 2005: 1 2)

Diese sehr weit gefasste Annäherung, die sowohl Lese- als auch Schreibaktivitäten umfasst, lässt noch offen, um welche Art von Texten es sich handelt und welche spezifischen Kompetenzen dabei benötigt werden. Ein engeres Verständnis von Textkompetenz formuliert demgegenüber Feilke (2012), auf dessen Konzept wir uns im Folgenden beziehen möchten: Er versteht sie als bloß eine von drei Dimensionen von Schreibfähigkeit, die sich ferner aus einer sozialkognitiven (psychologische Dimension des schreibenden Individuums) und einer schreibstrategischen Kompetenz (Schreibroutinen wie beispielsweise das Planen des Schreibprozesses) zusammensetzt (vgl. Feilke 2012: 8). Textkompetenz, als dritte Dimension, stellt dieser Auffassung zufolge eine „performative Kompetenz“ (ebd.: 10, Herv. d. V.) dar und beschreibt die Fähigkeit, Texte nach funktionalen

3 Dieser Beitrag ist im Kontext des SNF Projektes „Universitäre Lern Kommunikation in virtuel len Räumen“ (Laufzeit: 1.4.2012 bis 31.12.2016) entstanden (www.ds.uzh.ch/lernkommunika tion), das am Deutschen Seminar der Universität Zürich am Lehrstuhl von Prof. Dr. Heiko Hausendorf situiert ist. Wir danken Heiko Hausendorf, Carol Suter Tufekovic und zwei anony men GutachterInnen für kritische Kommentare zu früheren Versionen dieses Textes. 
Kriterien zu konstituieren. Diese Fähigkeit umfasst das „prozedurale Metawissen“ (ebd.) darüber, wie bestimmte sprachliche Kompositionselemente $\mathrm{zu}$ einem Textganzen zusammenzusetzen sind.

Im Zusammenhang mit Textkompetenz wiederum spielen Textroutinen eine zentrale Rolle. Damit sind sprachliche Kompetenzen gemeint, die wir uns im Verlauf unserer Lese- und Schreibbiographie aneignen. Sie beziehen sich vorrangig auf das Wissen, welche sprachlichen Konstruktionen existieren und wie diese bei der Herstellung eines Textes situativ adäquat und funktional eingesetzt werden können. Textroutinen können verstanden werden als „textkonstituierende sprachlich konfundierte literale Prozeduren, die jeweils ein textliches Handlungsschema (Gebrauchsschema) und eine saliente Ausdrucksform (Routineausdruck) semiotisch koppeln“ (Feilke 2012: 11). Oder anders ausgedrückt: Textroutinen sind rekurrente sprachliche Konstruktionen, für die eine identifizierbare Verbindung von Funktion und Form charakteristisch ist (vgl. Steinseifer 2010: 94). Bei der Textproduktion fungieren solche Textroutinen als „sprachliche Werkzeuge“ (Feilke 2012: 11), um spezifische Anforderungen schriftsprachlichen Handelns angemessen zu erfüllen. Dies zeigt sich auch in den von uns untersuchten Daten, wenn die Beteiligten spezifische saliente Formulierungsroutinen verwenden, um Arbeitsprozesse $\mathrm{zu}$ initiieren. Solche Formen routinisierten Schreibens sehen wir deshalb im Kontext dialogischen Schreibens als wichtigen konstitutiven Bestandteil der sprachlichen Bearbeitung nicht nur der von uns thematisierten Arbeitsinitiierung, die eine (zentrale) Teilaufgabe kollaborativen Arbeitens darstellt, sondern wir verstehen das routinisierte Schreiben allgemein als konstitutiv für die komplexe kommunikative Praxis des aufgabenorientierten kollaborativen Schreibens.

Aufgrund ihres wiedererkennbaren Form-Funktions-Zusammenhangs verweisen Textroutinen im Sinne von „Kontextualisierungshinweisen“ (Auer 1986) überdies reflexiv auf bestimmte Kontexte, in denen sie sinnhaft zur Anwendung kommen (vgl. Feilke 2012: 18-19). Routinebildung dient folglich nicht nur der „Entlastung von Formulierungsarbeit“ (ebd.: 6), sondern auch der Etablierung wechselseitiger Erwartungen. Besonders relevant wird dies im Rahmen dialogischen Schreibens. Hier spielen die Erwartungen der TeilnehmerInnen und deren Erfüllung oder Enttäuschung eine zentrale Rolle für das Gelingen oder NichtGelingen der Kommunikation, da der Austausch aus mindestens zwei aufeinander bezogenen Schreib- und mindestens einer Leseaktivität von mindestens zwei Beteiligten besteht. ${ }^{4}$ Oder anders ausgedrückt: Person A verfasst einen Text, der

4 Dies im Gegensatz zu monologischen Texten, die dadurch charakterisiert sind, dass Schreibende sich schriftsprachlich an Lesende wenden, die den Text rezipieren, selbst jedoch keine schriftliche Reaktion darauf verfassen. 
von Person B rezipiert wird, und auf dieser Basis verfasst B nun einen zweiten Text, der auf den ersten reagiert und sich an A richtet, usw. Dialogische Schriftlichkeit zeichnet sich daher dadurch aus, dass im Text kommunikative Zugzwänge etabliert werden, durch die Antworttexte der Lesenden erwartbar gemacht werden. Ähnlich nennt Günthner (2011) für die SMS-Kommunikation folgende dialogische Eigenschaften:

SMS Beiträge bilden eine dialogisch ausgerichtete Kommunikationsform, die sich durch eine sequenzielle Abfolge von Mitteilungen bzw. Dialogzügen auszeichnet, welche am zeitlichen Davor ausgerichtet sind, konditionelle Relevanzen für die Folgezüge setzen und sich mit dem jeweils spezifischen Rezipientendesign am Gegenüber orientieren: Die SMS Mitteilung ist somit das „gemeinsame Territorium“ von ProduzentIn und RezipientIn. (Günthner 2011: 30) ${ }^{5}$

Diese Eigenschaften rücken Kommunikationsformen wie SMS-, Foren- oder E-Mail-Kommunikation auf den ersten Blick in direkte Nähe zur Face-to-FaceInteraktion. Wir ziehen für Schriftkommunikation jedoch den Terminus „dialogisch“ der Bezeichnung „interaktiv“ vor, da wir letztere gemäß der systemtheoretischen Kommunikationsauffassung von Luhmann (1984) der Kommunikation unter Anwesenden vorbehalten wollen (vgl. dazu Hausendorf 2003; Hausendorf et al. 2015): Bei der Interaktion zwischen Anwesenden besteht Wahrnehmungswahrnehmung, d.h. die Beteiligten sind bereits während des Sprechens über Reaktionen der anderen Beteiligten orientiert und passen ihre Äußerungen noch während ihrer Genese laufend an diese Reaktionen an. Im Gegensatz dazu

wird die Produktion eines SMS Beitrags vom angesimsten Rezipienten nicht ,miterlebt‘, sondern erst dann wahrgenommen, wenn diese auf seinem Handy bemerkbar eingetroffen ist und rezipiert wird. [...] Folglich kann A erst dann wissen, ob ihre auf Wechselseitigkeit angelegte Kommunikation erfolgreich war, wenn B reagiert. (Günthner 2011: 16)

Mehr noch: Ob und wie der eigene Beitrag rezipiert wurde, erfahren Schreibende erst, wenn sie selbst wieder die Antwort lesen. Sie müssen also bereits ihren eigenen Beitrag diesen Unwägbarkeiten anpassen (vgl. Lindemann et al. i. Dr.). Dialogisches Lesen und Schreiben ist folglich grundlegend anders als die Interaktion unter Anwesenden.

Zusammenfassend sehen wir, dass dialogische Schriftkommunikation sich in ihren Bedingungen sowohl von Face-to-Face-Interaktion als auch von monologischem Schreiben unterscheidet. Erfolgreiches Kommunizieren in

5 Anders als Günthner (2011) nehmen wir im Folgenden den weiten Bachtin'schen Begriff von Dialogizität (Bachtin 1981) aus unseren Überlegungen aus, demzufolge jeder Text dialogisch ist, der sich intertextuell auf einen anderen bezieht. 
schriftdialogischen Zusammenhängen benötigt für diese besondere Kommunikationssituation spezifisches Wissen um passende und wirksame Textstrukturen und deren routinisierte Anwendung, kurz: Es erfordert dialogische Textkompetenz.

Die dafür notwendigen Textroutinen werden jedoch häufig nicht von sich aus beherrscht, sondern sie müssen zunächst erlernt und eingeübt werden. Denn zum einen können die in der Face-to-Face-Interaktion erprobten kommunikativen Verfahren nicht immer unverändert auf schriftliche Dialoge übertragen werden; zum anderen können Schreibende auch nicht einfach auf bekannte Textmuster monologischer Texte zurückgreifen. Bislang fanden diese Erfordernisse wenig Aufmerksamkeit in der Schreibforschung, sie sind empirisch und theoretisch kaum untersucht. An dieser Stelle setzen wir mit den folgenden Analysen an.

\section{Forenkommunikation auf einer Lernplattform - Anmerkungen zu den Daten}

Den Analysen dieses Beitrags liegen natürliche schriftsprachliche Daten aus Forenkommunikation zugrunde, die im Rahmen zweier einsemestriger E-Learning-Seminare entstanden und erfasst worden sind. ${ }^{6}$ Das didaktische Szenario dieser Blended-Learning-Veranstaltungen, bei denen Präsenzsitzungen mit Online-Lernen verknüpft werden, basiert auf dem Prinzip des „Forschenden Lernens“ (vgl. z. B. Huber 2004; Reiber 2007: bes. 8f.). ${ }^{7}$ Die Aufgabe der Studierenden bestand in diesen Seminaren darin, in Arbeitsgruppen von drei bis vier Personen, unterstützt durch eine Tutorin/einen Tutor, ein eigenes gesprächsanalytisches Forschungsprojekt $\mathrm{zu}$ planen, durchzuführen und abschließend die erarbeiteten Resultate zu präsentieren.

Für die gemeinsame Arbeit stand jeder Gruppe ein virtueller Arbeitsraum auf einer Online-Lernplattform zur Verfügung, der nur den Mitgliedern der jeweiligen Gruppe zugänglich war. Dort konnten die Studierenden auf

\footnotetext{
6 Das Gesamtkorpus, das in zwei Lehrveranstaltungen mit je 1112 Studierenden, drei TutorInnen und zwei Dozierenden entstand, umfasst 1490 Postings (103.538 Wörter) aus sechs verschiedenen Foren sowie Wikis und Aufgabenlösungen der Studierenden (Text Dokumente, Audio /Videodateien). Die untersuchte Lehrveranstaltung „gi Gesprächsanalyse interaktiv“ wurde am Deutschen Seminar der Universität Zürich am Lehrstuhl von Prof. Dr. Heiko Hausendorf konzipiert und wird regelmäßig für Studierende angeboten.

7 Vgl. Kesselheim/Lindemann (2010; 2012) zum didaktischen Szenario und zum Aufbau der Lernplattform.
} 
verschiedene Kommunikationsformen wie Forum, Chat und Wiki sowie eine virtuelle Ablage zurückgreifen, in die Dateien hochgeladen werden konnten. Alle Gruppen nutzten für die Arbeitsorganisation fast ausschließlich das Forum, das deshalb Gegenstand dieses Beitrags ist.

Über das ganze Semester hinweg hatten die Teilnehmenden klar definierte Arbeitsaufgaben sowie vorgegebene Abgabefristen einzuhalten und mussten daher oft unter Zeitdruck zusammenarbeiten. Dies stellte, ebenso wie die Bedingungen asynchroner Schriftkommunikation, hohe kommunikative und kooperative Anforderungen an die Beteiligten: Um die gestellten Aufgaben fristgerecht einreichen zu können, waren die Studierenden darauf angewiesen, zeitnahe Reaktionen auf ihre Postings zu erhalten. Um unnötige „Extraschleifen“ zu vermeiden, war es dabei wichtig, dass möglichst wenige Missverständnisse entstanden, dass also der Austausch schnell und reibungslos vonstattenging. Für eine effiziente Bearbeitung der Aufgaben war es zudem notwendig, die Arbeit (sinnvoll) untereinander aufzuteilen und sich bei der Bearbeitung abzustimmen. Dafür war kooperatives Kommunizieren wichtig, um Unstimmigkeiten in der Gruppe oder Motivationsverlust einzelner Beteiligter zu vermeiden. Ein kommunikativ und kooperativ besonders anspruchsvoller und zugleich für den Arbeitsprozess zentraler Moment ist dabei die hier untersuchte Initiierung eines neuen Arbeitsschritts.

In den meisten Fällen wird in den von uns untersuchten Daten zur Initiierung einer neuen Aufgabe zugleich auch ein neuer Thread eröffnet. Unsere Analysen der Initiierung von Arbeitsschritten basieren daher auf den initialen Beiträgen aller 101 Threads der sechs gruppeninternen Diskussionsforen unseres Gesamtkorpus. Dafür wurden sowohl die 101 Initialpostings als auch die jeweils darauf folgenden Postings untersucht. Die Eröffnung eines neuen Threads stellt einen Schlüsselmoment in der Gruppenkommunikation dar, da dort für alle Beteiligten erkennbar ein neuer Kommunikationsstrang eröffnet und an exponierter Stelle platziert wird, wodurch der Autor oder die Autorin strukturierend in die laufenden Kommunikationsprozesse der Gruppe eingreift. Der bestehende Austausch wird unterbrochen, es wird ein konkurrierender Kommunikationsort geschaffen und ggf. der inhaltliche Austausch in eine neue Richtung gelenkt.

Der Initiierung von Arbeitsschritten kommt zum einen besondere Bedeutung $\mathrm{zu}$, weil es sich dabei um einen notwendigen Prozessabschnitt handelt, der die gemeinsame Arbeit erst ermöglicht, indem ein Anfang gesetzt und benannt wird. Zum anderen ist dieser Moment kommunikativ prekär - gerade in einer gleichberechtigen Gruppe, wo Anweisungen an die übrigen Beteiligten hinsichtlich der Arbeitsplanung als Bevormundung missverstanden werden können: In Teams, die nicht-hierarchisch organisiert sind, sind die Aufgaben der 
Gesprächsstrukturierung und der Arbeitsorganisation keinem Teammitglied institutionell zugewiesen. Um einen neuen Arbeitsschritt $\mathrm{zu}$ initiieren, muss ein Gruppenmitglied den anderen also ohne institutionelle Legitimation Anweisungen geben. Da der Vollzug dieser Aufgabe nicht durch eine institutionell zugewiesene Rolle abgestützt ist, muss er auf andere Weise legitimiert werden. Daher müssen die Beteiligten ihr Anliegen sprachlich auf eine Weise an die Gruppe herantragen, die eine konfliktäre Situation vermeidet und zugleich den anstehenden Arbeitsschritt erfolgreich anstößt.

Schreibende benötigen für diese Aufgabe sowohl kommunikative als auch kooperative Kompetenzen, die nicht nur die Kommunikationsform (hier die asynchrone Kommunikation in einem Forum) und den adressierten TeilnehmerInnenkreis berücksichtigen, sondern auch, welches Ziel mit der Kommunikation verfolgt wird (in diesem Fall die gemeinsame Durchführung einer Aufgabe bzw. eines Projektes). Je nach Kommunikationsziel können sich dabei bestimmte kommunikative Verfahren als förderlich, andere als eher hinderlich erweisen.

Um der asynchron-schriftbasierten und zugleich dialogischen Qualität dieser Daten gerecht zu werden, stützen wir uns bei der Analyse zum einen auf (einzelne) grundlegende Konzepte der Konversationsanalyse (vgl. z. B. Sacks et al. 1974), die insbesondere für die Analyse der dialogisch organisierten Struktur schriftlicher Kommunikationen von großer Erklärungskraft sind. Dies gilt, obschon schriftliche Kommunikation grundlegend andere Konstitutionsbedingungen hat als Face-toFace-Interaktion (vgl. dazu die Überlegungen in Hausendorf et al. 2015). Deshalb sollten u. E. einzelne für mündliche Kommunikation etablierte Konzepte nicht ohne sorgfältige theoretische und methodische Reflexion auf schriftsprachliche Kontexte angewandt werden. ${ }^{8}$ Zum anderen greifen wir auf neuere Überlegungen der Textlinguistik zurück (vgl. z. B. Roth/Spitzmüller 2007; Fix 2008; Hausendorf/ Kesselheim 2008).

\section{Routinisiertes Schreiben bei der Aufgabeninitiierung}

Versteht man das rein schriftbasierte kooperative Arbeiten, wie wir es in unseren Daten vorfinden, als eine komplexe (Schreib-)Praxis, dann kann eine

8 Zur Frage der Übertragbarkeit solcher Konzepte auf schriftbasierte dialogische Kommunikation vgl. z. B. Baym 1996; Garcia/Jacobs 1999; Wolff 2006; Hutchby/Tanna 2008; Anderson et al. 2010. 
Arbeitsinitiierung als (zentrale) Teilaufgabe innerhalb dieser komplexen Praxis verstanden werden.

Postings, die einen Arbeitsschritt initiieren, sind üblicherweise so strukturiert, dass sie drei grundlegende kommunikative Aufgaben bearbeiten: Erstens wird begründet, weshalb das Posting und die Eröffnung eines neuen Threads überhaupt nötig sind (Account, vgl. Garfinkel 1967), zweitens wird ein Zugzwang (vgl. Sacks 1992: I, 673) für die LeserInnen etabliert, nun selbst aktiv zu werden, und drittens wird eine eigene (Vor-)Leistung dokumentiert. Diese wiederkehrenden Bestandteile arbeitsschrittinitiierender Postings sind fast ausnahmslos entweder in der Reihenfolge „Account-Zugzwang-Vorleistung“ oder „AccountVorleistung-Zugzwang“ angeordnet.

Initialpostings machen also erstens, fast immer einleitend, erkennbar, warum an dieser Stelle ein neuer Thread und ein neuer Arbeitsschritt initiiert werden. Sie formulieren einen sogenannten Account, der für andere Teilnehmende nachvollziehbar macht, welche Aktivität gerade durchgeführt wird und ggf. warum dies gerade jetzt getan wird. Damit machen sie diese Aktivität „visibly-rational-andreportable-for-all-practical-purposes“ (Garfinkel 1967, vii) und liefern eine Antwort auf die Frage „why that now?“ (vgl. Schegloff/Sacks 1973).

Dass mit einer Threaderöffnung stets auch das eigene Schreiben legitimiert wird, wird im Kontext schriftlicher kooperativer Arbeit unter Berücksichtigung des zweiten Bestandteils der thread-initiierenden Postings verständlich. Denn mit diesem werden Folgeerwartungen für nachfolgende Beiträge geschaffen, indem erwartbare weitere kommunikative Schritte der anderen Teilnehmenden projiziert werden. Dies wird im Fall der arbeitsinitiierenden Postings dadurch erreicht, dass zwei Arten von Zugzwängen, sog. konditionelle Relevanzen (conditional relevance, Schegloff 1968), gegenüber den anderen Gruppenmitgliedern etabliert werden. Diese Zugzwänge machen zum einen eine Antwort relevant (kommunikativer Zugzwang) und zum anderen wird die Mitarbeit der anderen Teilnehmenden erwartbar gemacht (kooperativer Zugzwang). Daraus erklärt sich, dass nicht nur der Anstoß gemeinsamer Arbeit eine „Zumutung“ für das Gegenüber darstellt, sondern bereits jegliche Textproduktion, denn: Im Kontext einer Gruppenarbeit muss jeder Text von den übrigen Beteiligten gelesen werden, er muss beantwortet werden - denn nur so wird der Leseprozess für die anderen Gruppenmitglieder überhaupt erkennbar (vgl. Hausendorf et al. 2015; Lindemann et al. i. Dr.) -, und im Fall einer Arbeitsinitiierung muss zusätzlich auch noch eine Aufgabe im Rahmen der Gruppenarbeit übernommen werden. Aufgabeninitiierende Postings produzieren somit gleich mehrfache Zugzwänge für die übrigen Beteiligten, und zwar wie beschrieben sowohl auf kommunikativer als auch auf kooperativer Ebene; sie bauen einen (Erwartungs-)Druck auf, der mit dem initial platzierten Account legitimiert wird. 
Als dritte kommunikative Aufgabe tritt in arbeitsinitiierenden Postings die Dokumentation einer erbrachten Leistung hinzu. Die Studierenden machen ihre eigene Leistung zugänglich, indem sie ihrem Beitrag entweder ein Textdokument anhängen, einen entsprechenden Text direkt im selben Posting platzieren oder aber auf einen anderen virtuellen Ort (Wiki, Ablageordner o. a.) verweisen, wo ihre Vorleistung dokumentiert ist. Sie zeigen dadurch an, dass sie selbst bereits eine gewisse Leistung für die Bearbeitung der anstehenden Aufgabe erbracht haben. Damit verstärken sie sowohl den kommunikativen als auch den kooperativen Zugzwang für die übrigen Beteiligten.

Für initiierende Postings lassen sich bei der Bearbeitung dieser drei kommunikativen Aufgaben - der Formulierung von Account, Projektion und Vorleistung - jeweils wiederkehrende (Text-)Strukturen im Sinne von Textroutinen erkennen. Im Folgenden zeigen wir exemplarisch zwei Verfahren, mit denen die für dialogische Schriftkommunikation relevante kommunikative Aufgabe der Initiierung eines Arbeitsschritts in einem Online-Forum routinisiert realisiert werden kann. Beim ersten Initiierungsverfahren übernehmen Schreibende eine koordinierende Funktion im anschließend gemeinsam bewältigten Arbeitsprozess, während Schreibende, die sich des zweiten Verfahrens bedienen, durch ihre inhaltliche Vorarbeit für die übrigen Gruppenmitglieder eine starke konditionelle Relevanz etablieren, die Aufgabe ohne die Autorin/den Autor des Initialpostings zu Ende zu führen.

\subsection{Initiierungsverfahren I: Zusammenarbeit anstoßen}

Ein erstes Initiierungsverfahren, mit dem ein Gruppenmitglied die übrigen Gruppenmitglieder zur Aufnahme der gemeinsamen Arbeit veranlassen kann, besteht darin, primär auf die institutionell vorgegebene Notwendigkeit eines nächsten Arbeitsschritts aufmerksam zu machen, der von der Gruppe bis zu diesem Zeitpunkt noch nicht (explizit) angegangen worden ist. Damit tritt dieses Gruppenmitglied in die Rolle einer Koordinatorin/eines Koordinators, die/der sich zu Beginn einer neuen Arbeitsphase um die Organisation der gemeinsamen Arbeit bemüht.

Abbildung 1 zeigt ein Beispiel für ein solches Eröffnungsverfahren. Die Autorin, Christina, ${ }^{9}$ verweist einleitend auf eine Frist, die die Gruppe für die Überarbeitung von Textdokumenten einzuhalten hat. Danach geht sie inhaltlich auf verschiedene Unsicherheiten ein, die im Vorfeld der gemeinsamen Weiterarbeit an den Dokumenten geklärt werden müssen. Abschließend

9 Bei allen Namen handelt es sich um Pseudonyme. 


\begin{tabular}{|l|l|} 
Überarbeitung & \multicolumn{1}{c|}{ Erstellt am 03.06.11 15:47 } \\
Christina Brunner & $\begin{array}{l}\text { Wallo zusammen! } \\
\text { ihr, sind davon betroffen? Ich denke, dass wir beispielsweise das Transkript so } \\
\text { belassen können (höchstens auf Flüchtigkeitsfehler überprüfen). Und bei den } \\
\text { anderen Dokumenten bin ich mir nicht sicher, ob gemeint ist, dass wir auch } \\
\text { inhaltlich was ändern sollen, oder nur formal. } \\
\text { Ab dem 7. Juni kann ich mich leider nicht mehr aufs gi-Seminar konzentrieren, } \\
\text { da dann mein Block Rechtsprüfungen beginnt und ich gar nicht die Möglichkeit } \\
\text { habe, ins Internet zu gehen. Ich kann ja sonst den ersten Schliff in den nächsten } \\
\text { Tagen übernehmen. }\end{array}$ \\
\hline Löschen Editieren Antworten mit Zitat Antworten ohne Zitat
\end{tabular}

Abbildung 1: Initiierungsverfahren I: „Zusammenarbeit anstoßen“.

kündigt sie ihre bevorstehende Abwesenheit an und schlägt Möglichkeiten einer ausgeglichenen Arbeitsteilung vor: ${ }^{10}$

In Forenbeiträgen dieser Art werden typischerweise die drei bereits genannten kommunikativen Aufgaben routinisiert bearbeitet: Die Postings geben erstens einen impliziten Hinweis auf den Anlass des Postings in thread-initialer Position (Account), fordern zweitens (kommunikative) Anschlusshandlungen ein (Projektion) und präsentieren drittens einen aufgabenbezogenen Input in Form inhaltlicher Überlegungen und/oder Ideen zur weiteren Planung der Gruppenarbeit (Vorleistung).

In der Regel bildet der Account den einleitenden Teil des Textes nach der (optionalen) Adressierung. Dabei wird stets auf die institutionell vorgegebenen Abgabefristen (bis zum 8. Juni) oder Vorgaben (Wir können ja [...] die Dokumente noch überarbeiten) verwiesen. Der für diese Art von Posting musterhafte Hinweis auf eine von außen auferlegte institutionelle Verpflichtung kann im Kontext der Kommunikation innerhalb einer nicht hierarchisch organisierten Gruppe als Rechtfertigung für das eigene „Vorpreschen“ verstanden werden. Das in diesem Beispiel verwendete Modalverb ,können` verweist zwar zunächst auf eine Möglichkeit der Überarbeitung anstelle einer strikten Vorgabe. Insofern die Gruppe eine weniger gute Note für ihr Portfolio riskiert, wenn sie trotz vorhandener Kritik die Dokumente nicht überarbeitet, hat die optionale Überarbeitung hier jedoch durchaus den Charakter einer institutionellen Vorgabe.

10 Diesen letzten Absatz nehmen wir aus der folgenden Analyse aus, da dort von der Autorin nicht die kommunikative Aufgabe der Initiierung, sondern eine weitere Problematik bearbeitet wird. 
Mit der Verwendung der ersten Person Plural (,Wir können“) zeigt die Autorin außerdem an, dass sie sich selbst als Teil der von den externen Vorgaben betroffenen Gruppe verstanden wissen möchte. Damit positioniert sie sich als Mitglied der Gruppe und vermeidet eine eindeutige Selbstpositionierung als Koordinatorin, die den anderen von einer übergeordneten Stellung aus Aufträge zuweist (vgl. zur Selbst- und Fremdpositionierung grundlegend Hollway 1984; Davies/Harré 1990; Lucius-Hoene/Deppermann 2004). Auch andere AutorInnen verwenden ähnliche Formulierungen, die insgesamt als Account für diese Form der Threaderöffnung salient sind:

[...] schon in einer Woche müssen wir den nächsten Schritt durchgeführt haben.

(Sebastian, „Schritt 3: Daten erheben“, 25.03.2010, 21:43)

Wir müssen ja bis morgen ein Erhebungsszenario hochladen [...] .

(Marion, „Datenerhebung“, 31.03.2010, 13:20)

Die letzte Präsenzsitzung steht an. Wir sollen hier unsere Datenanalyse sowie den forschungsverlauf präsentieren.

(Melanie, „Präsentation“, 25.05.2011, 09:58)

Bis Donnerstag müssen wir schon wieder einen ersten Entwurf bereitstel len.

(Anna, „Interpretation der Daten“, 11.05.2010, 09:29)

Für den Account werden durchgängig Modalverben (müssen, sollen, können) eingesetzt, die anzeigen, dass es sich um eine der Gruppe durch die Institution auferlegte Pflicht bzw. eine institutionell zugestandene Handlungsoption handelt, sowie die erste Person Plural, die die AutorInnen in den von dieser Pflicht betroffenen Personenkreis einschließt. Zudem wird durch den (mehrheitlich initialen) Verweis auf eine Frist, oft verstärkt durch das Temporaladverb schon, die Dringlichkeit des Anliegens betont und damit zugleich indirekt gerechtfertigt, dass in einer Gruppe mit gleichberechtigten Mitgliedern unaufgefordert die Koordinationsaufgabe übernommen und den übrigen Beteiligten (Lese-) Arbeit aufgebürdet wird. Außerdem wird im Threadtitel (s. Abbildung 1, links oben im Postingrahmen) ${ }^{11}$ häufig die anstehende Aufgabe genannt (hier: „Transkribieren“), oft sogar mit der identischen Bezeichnung wie im offiziellen Semesterprogramm (z. B. „Transkribieren“, „Videoaufnahmen“ etc.). Auch dies

11 Um einen neuen Thread zu eröffnen, muss die Autorin oder der Autor ein Posting verfassen und diesem einen Titel geben. Dieser Titel in der Betreffzeile des ersten Postings wird auto matisch als Threadtitel übernommen. 
ist ein deutlicher Hinweis auf die Rückbindung an die institutionellen Vorgaben als Account für die Threaderöffnung und die Arbeitsinitiierung.

Dass sich die Schreibenden bei dieser Form der Aufgabeninitiierungen jeweils als Teil der Gruppe darstellen, zeigt sich auch in der zweiten Textroutine, der Projektion. In Beispiel 1 (s. Abbildung 1) initiiert die Autorin durch die Frage nach der Meinung der anderen (Welche meint ihr [...] ?) eine gemeinsame Diskussion in der Gruppe und projiziert damit die Kooperation in dieser Angelegenheit. Grundsätzlich zeigt die Autorin durch die Platzierung dieser Frage als Einleitung ihrer eigenen Überlegungen, dass sie nicht darauf hinwirkt, die Beantwortung auf die anderen Teammitglieder abzuwälzen. Vielmehr signalisiert sie damit, dass sie davon ausgeht, sich am weiteren Austausch zu beteiligen. Neben diesem inhaltlichen und strukturellen Hinweis nutzen andere AutorInnen grammatische Signale, indem sie häufig Formulierungen in der ersten Person Plural zur Betonung der gemeinsamen Weiterarbeit verwenden:

Wie gehen wir jetzt weiter vor?

(Christina M., „Transkription“, 27.04.2011, 17:28)

Wie gehen wir nun weiter vor?

(Margret, „Daten erheben“, 08.03.2011, 21:25)

wie sollen wir beim transkribieren vorgehen?

(Christine B., „Transkribieren“, 26.04.2010, 10:09)

Ganz im Sinne eines weiteren Zurücknehmens einer potenziell dominierenden Rolle der Autorin leitet diese in Beispiel 1 anschließend Überlegungen zur weiteren Arbeitsplanung mit zahlreichen Unsicherheitsmarkierungen ein ( Ich denke; bin ich mir nicht sicher). Ihre Vorschläge präsentiert sie dementsprechend nicht als fertig ausformuliertes Produkt, sondern im Sinne eines Abwägens von Vorüberlegungen für die gemeinsame Arbeit (beispielsweise; ob gemeint ist; oder nur formal).

In vielen anderen Postings dieses Initiierungsverfahrens finden sich in diesem planenden Teil zudem Hedges oder Konjunktive, die dem Eindruck entgegenwirken, die AutorInnen würden sich von der Gruppe abheben und sich als Mitglied mit mehr (kommunikativen) Rechten positionieren:

Ich würde sagen, wir befolgen den Rat von Martin

(Kira, „Überarbeitung Entwurf“, 20.03.2010, 14:04)

Ich wäre dafür, dass die Aufnahme nicht von einer Person gemacht wird, die die WG kennt, [...]

(Marion, „Datenerhebung“, 31.03.2010, 13:20) 
An sonsten würde ich vorschlagen, dass wir es aufteilen, [...] .

(Melanie, „Präsentation“, 25.05.2011, 09:58)

Und ich finde, für eine erste, grobe Phase ist das vielleicht gar nicht so schlecht.

(Christina M., „Transkription“, 27.04.2011, 17:28)

Solche Überlegungen zum weiteren Vorgehen finden sich durchgängig in (Initial-)Postings dieser Art. Es scheint also notwendig $\mathrm{zu}$ sein, dass die Autorin/der Autor den nächsten Schritt im Arbeitsprozess bereits angegangen ist, um eine gemeinsame Aufgabe auf diese Weise anstoßen zu können. Dies deutet darauf hin, wie wichtig es ist, sich an diesem Punkt der gemeinsamen Arbeit selbst als Teil der Gruppe zu positionieren und die Rolle als KoordinatorIn durch das Ankündigen der eigenen Mitarbeit wieder zurückzunehmen. Zudem erwirkt sich die Autorin im Kontext des kooperativen Arbeitens durch ihre Vorleistung das Recht, die anderen nicht nur in einen kommunikativen Zugzwang zu bringen, d. h. eine Antwort erwartbar zu machen, sondern auch einen kooperativen Zugzwang aufzubauen, d. h. eine Beteiligung an der gemeinsamen Arbeit einzufordern: Indem sie etwas zur Gruppenarbeit beiträgt und ankündigt, an weiteren Schritten teilzunehmen, regt sie die übrigen Gruppenmitglieder zum Mitmachen an und dazu, auf diese Weise ihrerseits das Gleichgewicht von Geben und Nehmen wieder herzustellen.

\subsection{Initiierungsverfahren II: Weiterarbeit einfordern}

Eine weitere Möglichkeit, wie ein Gruppenmitglied das Initiieren eines neuen Arbeitsschritts gestalten kann, besteht darin, diesen nicht nur anzuregen und vorzubereiten, sondern substantielle Schritte bereits selbst zu leisten und durch diese konkrete inhaltliche Leistung die übrigen Gruppenmitglieder zur Fortführung oder sogar zum Abschluss der Arbeit anzuhalten. Die Autorin oder der Autor übernimmt hier also keine Koordinationsaufgabe auf organisatorischer Ebene (s.o. Abschnitt 4.1), sondern leistet einen inhaltlichen Beitrag, den die übrigen Gruppenmitglieder weiterführen oder abschließen sollen. Damit ist dieses Initiierungsverfahren an einem anderen Ort innerhalb des kollaborativen Arbeitsprozesses zu situieren: Beteiligte initiieren hier vorrangig die Durchführung der inhaltlichen Aufgabenbearbeitung, während bei dem Initiierungsverfahren „Zusammenarbeit anstoßen“ die Planung des gemeinsamen Vorgehens im Vordergrund steht. Entsprechend wird das Verfahren „Weitarbeit einfordern“ auch sprachlich anders realisiert.

Abbildung 2 zeigt ein Beispiel für dieses kommunikative Verfahren. Hier wird ein Textentwurf präsentiert, auf den die Autorin im Postingtext verweist 
und auf dessen Grundlage sie weitere Beiträge der Gruppe einfordert: Die Autorin, Anna, berichtet einleitend, dass sie einen Entwurf für einen Text formuliert habe, den die Gruppe im Zuge des nächsten Arbeitsschritts einreichen muss. Den Entwurf selbst fügt sie in Postskriptum-Position ganz am Ende des Postings ein. Nach dem Einstieg bittet sie um Einschätzungen zum Text und fordert die Gruppe auf, nun ihrerseits die Ausformulierung des Textes zu übernehmen:

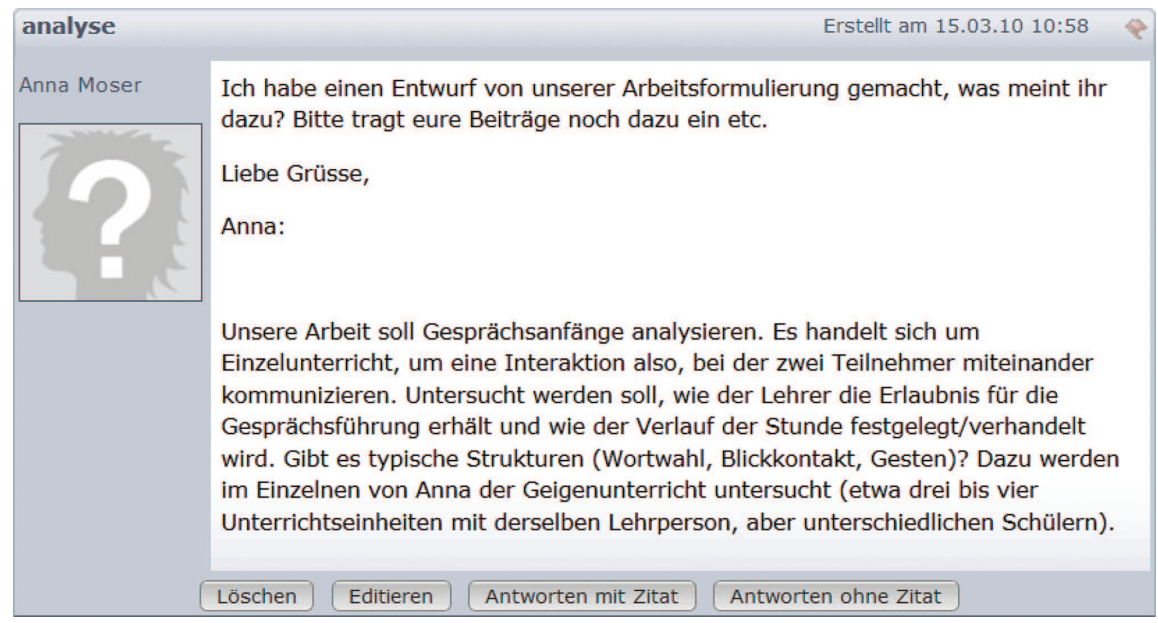

Abbildung 2: Initiierungsverfahren II: „Weiterarbeit einfordern“.

Auch hier lassen sich dieselben drei routinisiert bearbeiteten kommunikativen Aufgaben ausmachen, die wir bereits für das Initiierungsverfahren „Zusammenarbeit anstoßen“ beschrieben haben: Ein initialer Account, die Projektion in Form expliziter Einforderungen von Anschlusshandlungen sowie das Textprodukt, d.h. die Leistung selbst. Diese drei Bestandteile werden bei dem Initiierungsverfahren „Weiterarbeit einfordern“ jedoch in anderer Weise realisiert.

Als Account für den Anlass des Postings verweist die Autorin auf eine erbrachte Leistung (Ich habe einen Entwurf [...] gemacht). Während also in dem zuvor beschriebenen Eröffnungsverfahren ein Verweis auf institutionell vorgegebene Fristen als Erklärung für die Eröffnung eines neuen Threads dient (vgl. Abbildung 1), ist es hier der Hinweis auf die eigene bereits erbrachte Leistung. Dieser Account basiert also darauf, mit dem Verweis auf eine eigene notwendige - Leistung die „Zumutung“ zu legitimieren, die ein solches Posting 
mit sich bringt, denn: Es entstehen ja kommunikative und in diesem Falle auch gewichtige kooperative Zugzwänge für die übrigen Gruppenmitglieder, wenn die Autorin/der Autor des Initialpostings sich anschließend aus der weiteren Arbeit herausnimmt. Die Notwendigkeit dieses ersten inhaltlichen Arbeitsschritts wird dabei nicht mehr expliziert und mit Rückgriff auf die institutionell vorgegebenen Aufgaben und Fristen begründet, sondern als bereits bekannt vorausgesetzt. Durch den Hinweis auf die erbrachte Leistung positioniert sich die Autorin deutlich als Gruppenmitglied, das sich bereits aktiv für die Bearbeitung des gemeinsamen Projektes eingesetzt hat.

Diese kommunikative Aufgabe wird von anderen AutorInnen mit ähnlichen Formulierungen bewältigt: Die UrheberInnen der Leistung werden in der ersten Person Singular genannt, die Leistung selbst im Indikativ Perfekt als abgeschlossene Handlung präsentiert und das Produkt der Arbeit wird nicht nur im Postingtext, sondern häufig auch im Threadtitel genannt:

ich hab mal mit diesem blatt angefangen [...] .

(Julia, „Präsentation“, 22.05.2010, 17:02)

Habe noch das Erfassungsszenario hochgeladen!

(Lukas, „Erfassungsszenario“, 01.04.2010, 15:52)

Ich habe das vorgegebene Titelblatt sowie die Einverständniserklärungen [...] angefügt.

(Nils, „Die unendliche Geschichte“, 29.06.2010, 19:16)

Ich habe nun die Datenanalyse nochmals überarbeitet.

(Kira, „endgültige Abgabe Datenanalyse“, 17.05.2010, 19:58)

Ich habe jetzt mal verschiedene Daten im Ordner hochgeladen.

(Laura, „Portfolioordner“, 20.06.2010, 20:02)

Durch diese Formulierungen machen die AutorInnen deutlich, dass sie eine substantielle Leistung für die Gruppe erbracht haben. Damit wird die nachfolgende, ebenfalls routinisiert realisierte Aufforderung an die übrigen Gruppenmitglieder, die Arbeit fortzusetzen oder abzuschließen, legitimiert. Die Autorin nimmt sich aus dem folgenden Bearbeitungsschritt aus, indem sie die zweite Person Plural verwendet (was meint ihr dazu?) und stattdessen ihre Kommilitoninnen mittels Imperativ (Bitte tragt eure Beiträge noch dazu ein) explizit zu einer eigenen Leistung auffordert.

Sowohl durch die Frage als auch durch den Imperativ etabliert die Autorin gegenüber den übrigen Gruppenmitgliedern verschiedene Zugzwänge, nämlich zum einen auf kommunikativer Ebene in Form erwartbar gemachter Antwortpostings sowie auf Ebene der Kooperation, indem inhaltliche Beiträge 
gefordert werden. Diese Projektion wird in der Mehrzahl solcher Postings ähnlich formuliert:

Bitte scheut euch nicht, zu ändern und zu ergänzen;)

(Nils, „Schriftliche Präsentation“, 11.06.2010, 12:42)

Schauts euch doch kurz im Wiki [...] an, und korrigiert das, was euch nicht gefällt, oder unvollständig erscheint.

(Lukas, „Erfassungsszenario“, 01.04.2010, 15:52)

Bitte schaut es euch an und verbessert nach eigenem Gusto.

(Sebastian, „Endversion ,Daten erheben““, 08.04.2010, 00:28)

Schaut ihn euch unbedingt mal an und sagt mir, wenn was fehlt oder ich was weglassen soll oder was falsch ist!

(Kira, „endgültige Version PPP Vortrag“, 27.05.2010, 16:11)

Was meint ihr?

(Nils, „Die unendliche Geschichte“, 29.06.2010, 19:16)

Die Forderungen werden jeweils im Imperativ an die Gruppe gerichtet, gelegentlich durch Fragen im Indikativ in der zweiten Person Plural. Beide Formen machen in diesem Kontext deutlich, dass die AutorInnen sich aus der gemeinsamen Arbeit ausnehmen und diese an die anderen Gruppenmitglieder übergeben. Im Gegensatz zum zuvor erläuterten Initiierungsverfahren „Zusammenarbeit anstoßen“ werden hier keine Konjunktive verwendet, die den Aufforderungscharakter an die Gruppe mildern würden. Lediglich Abtönungspartikeln wie mal oder kurz schwächen den Imperativ ab. In einigen Fällen wird dabei zugleich durch die Verwendung von Partikeln wie unbedingt oder doch die Notwendigkeit einer Reaktion hochgestuft.

Legitimiert wird diese explizite Forderung an die anderen durch die eigene substantielle Leistung für die Gruppe. Diese Leistung in Form eines ersten Textentwurfs oder einer lediglich noch zu korrigierenden Version scheint notwendig und zugleich ausreichend, um die Verantwortung für die Weiterarbeit an die übrigen Gruppenmitglieder abzugeben. Es scheint dabei bedeutsam, dass die präsentierte Leistung nicht lediglich eine Sammlung erster Ideen ist (wie in dem unter 4.1 beschriebenen Verfahren), sondern als eigenständiges Produkt dargestellt wird, dessen Bearbeitungsstatus fast abgeschlossen ist und das vor der Fertigstellung lediglich von den übrigen Gruppenmitgliedern ergänzt, korrigiert oder bewertet werden kann oder soll. Im obigen Beispiel (s. Abbildung 2) befindet sich der Textentwurf in der Postskriptum-Position und ist durch mehrere Leerzeilen erkennbar abgetrennt von der Mitteilung an die KommilitonInnen; in der Mehrzahl der Fälle wird das Produkt sogar als Textdatei dem Posting angehängt. Durch 
dieses Vorgehen wird das Gewicht der eigenen Leistung zusätzlich betont, indem das Produkt nicht nur durch die Gliederung des Postings hervorgehoben wird, sondern selbst eine eigenständige neue Texteinheit bildet.

Unter der Perspektive einer ausgeglichenen Arbeitsbelastung entsteht beim Initiierungsverfahren „Weiterarbeit einfordern“ im Gegensatz zum Verfahren „Zusammenarbeit anstoßen“ aufgrund der umfassenden Arbeit der AutorInnen ein starkes Ungleichgewicht, so dass sie sich anschließend aus der Weiterarbeit ausnehmen können. Der Ausgleich zwischen Geben und Nehmen muss nun erst einmal durch Beteiligung der anderen Gruppenmitglieder wiederhergestellt werden. Während also bei beiden Initiierungsverfahren ein kommunikativer Zugzwang hinsichtlich einer schriftlichen Reaktion etabliert wird, unterscheiden sie sich bezüglich der etablierten kooperativen Zugzwänge: Im ersten Fall wird eine gemeinsame Diskussion über Vorgehensweisen und Inhalte angestoßen, während im zweiten Fall über diesen ersten Prozessschritt hinaus die Weiterarbeit an einem fortgeschrittenen Produkt in Auftrag gegeben wird.

\subsection{Routinisiertes Schreiben als Lernaufgabe}

Wo die Beteiligten in unseren Daten Arbeitsprozesse erfolgreich initiieren, handeln sie sehr routinisiert und gleichförmig hinsichtlich der Textstruktur. Jedoch finden sich auch Beispiele, in denen diese Strukturen nur partiell umgesetzt werden und wo im Zuge dessen Unregelmäßigkeiten im Kommunikations- und/ oder Kooperationsprozess zu beobachten sind.

Beispiel 3 zeigt eine partiell realisierte Variante des Initiierungsverfahrens „Zusammenarbeit anstoßen“: Account und Projektion werden zwar auf fast prototypische Weise umgesetzt, es werden aber keine eigenen ersten Überlegungen zum weiteren Vorgehen vorgebracht:

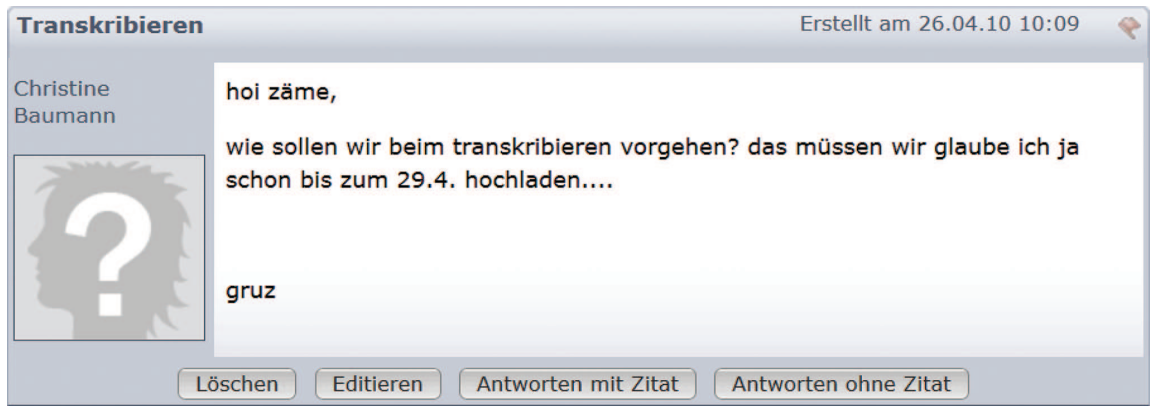

Abbildung 3: Partiell realisiertes Initiierungsverfahren „Zusammenarbeit anstoßen“. 
Die Autorin stößt die gemeinsame Arbeit durch die Frage an die Gruppe an, wie der nächste Arbeitsschritt organisiert werden soll. Im nächsten Satz begründet sie ihre Frage durch den Hinweis auf die institutionellen Vorgaben, denen die Gruppe Folge leisten muss, sowie durch den Verweis auf den daraus resultierenden Zeitdruck (die Transkription hat in den nächsten drei Tagen $\mathrm{zu}$ erfolgen). Eigene Ideen zur Arbeitsorganisation präsentiert sie nicht. Sie realisiert mit ihrem Posting also Projektion und Account, aber keine Vorleistung. Wie im darauffolgenden Posting sichtbar wird (Abbildung 4), scheint aus einem solchen „Fehlen“ einer üblicherweise realisierten Textroutine dieses Initiierungsverfahrens ein kommunikativer Bedarf zu resultieren, diese (Leer-)Position zu füllen. Entsprechend kann das folgende Beispiel als Versuch verstanden werden, auf das im Initialposting nur partiell realisierte Initiierungsverfahren $\mathrm{zu}$ reagieren:

\begin{tabular}{|c|c|}
\hline \multicolumn{2}{|c|}{ Re:Transkribieren } \\
\hline etra Schmidt & $\begin{array}{l}\text { hallo! } \\
\text { die zeit ist wirklich verdammt knapp..... - vor allem wenn wir unsere } \\
\text { Sequenzen nochmals anpassen müssen. Ich würde vorschlagen, dass wir } \\
\text { ihnen noch doch alle unsere Sequenzen zukommen lassen und überall ein } \\
\text { paar Sekunden zusätzlich dranhängen. } \\
\text { Dann teilen wir die Sequenzen nach Minutenzahl auf und transkribieren das } \\
\text { bis zum Donnerstagmorgen und geben bis Donnerstagabend gegenseitig } \\
\text { Rückmeldungen und laden dann alles gemeinsam hoch. Was haltet ihr } \\
\text { davon? } \\
\text { Und habt ihr verstanden, warum sie unsere Ausschnitte nicht so gut } \\
\text { fanden??... }\end{array}$ \\
\hline & $\begin{array}{l}\text { chen Editieren Antworten mit Zitat Antworten ohne Zitat } \\
\text { seitrag mit Antworten abtrennen Verschieben in anderes Thema }\end{array}$ \\
\hline
\end{tabular}

Abbildung 4: Vervollständigung des Initiierungsverfahrens „Zusammenarbeit anstoßen“ im Folge-Posting.

Zeitstempel und Benutzername im Postingrahmen (rechts und links oben) zeigen, dass der Vorschlag, wie die gemeinsame Transkriptionsarbeit geplant werden könnte (Ich würde vorschlagen), erst im darauffolgenden Beitrag eines anderen (!) Gruppenmitglieds gemacht wird. Eingeleitet wird das Posting zudem durch eine Bekräftigung der Zeitknappheit, und darüber hinaus fordert die Autorin die Gruppe mit einer neuerlichen Projektion zur Mitarbeit auf (Was haltet Ihr davon?). Account, Vorleistung und Projektion werden in diesem zweiten Posting somit - sechs Stunden nach dem ersten - musterhaft realisiert. 
Grundsätzlich scheint ein unvollständig realisiertes Initiierungsverfahren zwar nicht unbedingt kommunikative Probleme auszulösen, wie auch diese beiden Postings zeigen. Jedoch bedeutet die daraus resultierende „Extra-Schleife“ kommunikativen Mehraufwand und ist insofern im Kontext gemeinsamen Arbeitens als potentiell krisenhaft anzusehen, denn: Jede zusätzliche Kommunikationsschleife verzögert den Fortgang um ungewisse Zeit, da Foren-Kommunikation eine asynchrone Kommunikationsform ist und deshalb die Schreibenden nicht wissen können, wann ihr Beitrag gelesen und beantwortet wird (s.o. Abschnitt 2). Diese Verzögerung aufgrund einer nicht realisierten Routine ist also insbesondere in diesem kooperativen Arbeitszusammenhang, der mit knappen Fristen verbunden ist, riskant und wenig effizient.

\section{Diskussion und Ausblick}

Ausgehend von Beispielen aus einem studentischen Diskussionsforum haben wir zwei kommunikative Verfahren vorgestellt, die in einem kollaborativen Arbeitsund Lernszenario die Funktion übernehmen, die Bearbeitung einer neuen Aufgabe zu initiieren. Typisch für die von uns untersuchten Initiierungsverfahren ist, dass die Schreibenden die Aktivität des Initiierens zunächst begründen (Account), dass sie dann eine kommunikative Anschlusshandlung und inhaltliche Weiterarbeit der KommilitonInnen einfordern (Projektion) und dass sie schließlich einen ersten Input platzieren, der mit dem zu initiierenden Aufgabenschritt zusammenhängt (Leistung). Die Schreibenden realisieren diese Teilaufgaben sprachlich (und grafisch) musterhaft, greifen also auf Textroutinen zurück, die ihnen eine reibungslose Realisierung der kommunikativen Aufgabe „Arbeitsschritt initiieren“ im Rahmen des gemeinsamen Arbeitens ermöglichen (s. 4.1 und 4.2).

An rekurrenten Strukturen dieser Art lässt sich erkennen, dass auch dialogische Schriftlichkeit im Kontext kooperativen Arbeitens prozedurales TextWissen in Form von Routinen erfordert. Der Gebrauch von Textroutinen und damit Textkompetenz ist also nicht auf klassische monologische Texte beschränkt. Deshalb ist es unseres Erachtens wichtig, Textkompetenz-Modelle um die dialogische Dimension zu erweitern und in solchen Zusammenhängen von „dialogischer Textkompetenz“ zu sprechen.

Damit AutorInnen in schriftdialogischen kooperativen Zusammenhängen tatsächlich diejenigen Reaktionen erhalten, die sie anstreben, benötigen sie sowohl kooperative als auch kommunikative Kompetenzen: Sie müssen ihre Texte an die affordances (vgl. Gibson, 1979) der Kommunikationsform anpassen (die in den sich schnell entwickelnden Online-Kontexten häufig wechseln), und 
sie müssen - wie in Face-to-Face-Interaktion auch - den adressierten TeilnehmerInnenkreis und den Zweck der Kommunikation berücksichtigen. Dabei hilft den AutorInnen dialogische Textkompetenz in Form von (dialogisch) routinisiertem Schreiben.

Wie andere komplexe kommunikative Aufgaben auch, werden diese Verfahren von den Beteiligten jedoch nicht in jedem Fall einfach beherrscht; auch dialogische Textkompetenz muss erworben werden: Textroutinen, die aufgabenorientiertem und kooperativem dialogischem Schreiben zugrunde liegen, müssen zunächst in ihren kommunikativen und kooperativen Implikationen erkannt und ggf. gelernt und eingeübt werden, damit sie effizient zur Anwendung kommen können. In unseren Daten zeigt sich dies in zweierlei Hinsicht: Erstens darin, dass in Fällen, in denen die typische dreigliedrige Struktur der von uns beschriebenen Initiierungsverfahren nicht oder nur partiell realisiert wurde, kommunikative Missverständnisse oder Verzögerungen der Kooperationsprozesse die Folge sein konnten (s. 4.3). ${ }^{12}$ Zweitens wird zwar das Verfahren „Weiterarbeit Einfordern“ zumeist identisch in prototypischer Weise realisiert, für das Verfahren „Arbeit anstoßen“ liegen hingegen kaum Postings vor, die über alle drei Textroutinen hinweg prototypisch formuliert sind. Dies legt die Vermutung nahe, dass es sich bei dem Verfahren „Arbeit anstoßen“ um eine weniger bekannte und/oder schwieriger zu realisierende Routine handelt, die im Verlauf eines Semesters noch nicht vollständig gefestigt werden konnte.

Ausgehend von der Annahme, dass in Schule und Beruf dem Erwerb dialogischer Textroutinen und damit dialogischer Textkompetenz zunehmend größere Bedeutung zukommt, plädieren wir dafür, kooperatives Arbeiten in den neuen Medien empirisch vermehrt zu untersuchen und dabei Konzepte aus der Schreibforschung auf ihre Übertragbarkeit auf schriftdialogische Kommunikationszusammenhänge hin zu überprüfen und allenfalls zu erweitern. Zudem sollte die Fähigkeit, in kooperativen Arbeitskontexten dialogisch zielführend zu schreiben, schulisch gelehrt und gefördert werden. Denn auch in dialogischen Schreibzusammenhängen gilt, dass „die Fähigkeit, die sozial etablierten Formen angemessen zu verwenden, [...] entscheidend für die pragmatische Ein-Passung und Akzeptabilität von Texten“ (Steinseifer 2010: 92) ist.

12 Mit unseren Analysemethoden können wir zwar keine Aussage dazu machen, ob sich AutorInnen solcher Postings bewusst dafür entscheiden, ein Initiierungsverfahren nicht vollständig zu realisieren (z. B. keinen eigenen Vorschlag zur Organisation der Arbeit zu formu lieren, etwa deshalb, weil damit persönlicher Aufwand verbunden ist), oder ob ihnen die nötige dialogische Textkompetenz für die vollständige Bearbeitung des entsprechenden Verfahrens (noch) fehlt. Aber wir können feststellen, dass es für die Umsetzung kooperativer Vorhaben in schriftdialogischen Kontexten zielführend ist, alle drei beschriebenen, für die jeweiligen Initiierungsverfahren typischen Textroutinen (Account, Projektion, Leistung) einzusetzen. 
Es wäre also wichtig, dass AutorInnen so kompetent - im Sinne von geübt sind, dass sie diese Textroutinen beherrschen und dann situativ entscheiden können, ob sie sie einsetzen oder nicht.

Aufgabe von Schule und Universität wäre es dann, nicht mehr nur mit Blick auf monologische, sondern auch auf dialogische Textkompetenz „Hand zu bieten, um für die Bewältigung dieser kommunikativen Aufgaben adäquat gerüstet zu sein“ (Spiegel/Kleinberger Günther 2006: 198). Die Vermittlung von Inhalten sollte sich dabei nicht darin erschöpfen, abstrakte Regeln und Maximen zum dialogischen Schreiben zu erläutern, sondern die damit verbundenen Aufgaben, Probleme sowie deren mögliche Lösungen sollten in Form praxisbezogener Anwendungen gemeinsam erarbeitet werden (vgl. Lehnen/Jakobs 2003: 404). Deshalb wäre es wichtig, virtuelle Zusammenarbeit in Projektarbeiten erfahrbar zu machen, anstatt sie nur theoretisch zu thematisieren. Dafür besonders geeignet scheinen Lernszenarien, bei denen die Teilnehmenden auf das Gelingen der kommunikativen Prozesse tatsächlich angewiesen sind - wie beispielsweise in der Veranstaltung „gi - Gesprächsanalyse interaktiv“, aus der die hier untersuchten Daten stammen. Sie ermöglichen es den Lernenden, dialogische Textroutinen unter realistischen Bedingungen zu erproben. Erweitert man diese Lernszenarien didaktisch um eine Reflexion der kommunikativen Prozesse, können SchülerInnen und Studierende dabei unterstützt werden, sich dialogische Textkompetenz aktiv für unterschiedliche Kommunikationsaufgaben anzueignen.

\section{Literatur}

Anderson, Jeffrey F., Fred K. Beard \& Joseph B. Walther. 2010. Turn-taking and the Local Management of Conversation in Highly Simultaneous Computer-Mediated Communication. Language@Internet 7. Article 7.

Auer, Peter. 1986. Kontextualisierung. Studium Linguistik 19. 22-47.

Bakhtin [Bachtin], Mikhail M. 1981. The Dialogic Imagination: Four Essays. Ed. by Michael Holquist. Austin TX: University of Texas Press.

Baym, Nancy K. 1996. Agreements and Disagreements in a Computer-Mediated Discussion. Research on Language and Social Interaction 29. 315-345.

Davies, Bronwyn \& Rom Harré. 1990. Positioning: the discursive production of selves. Journal for the Theory of Social Behaviour 20(1). 43-63.

Feilke, Helmuth. 2012. Was sind Textroutinen? Zur Theorie und Methodik des Forschungsfeldes. In Helmuth Feilke \& Katrin Lehnen (eds.), Schreib und Textroutinen: Theorie, Erwerb und didaktisch mediale Modellierung, 1-31. Frankfurt a.M., Bern, New York: Lang.

Fix, Ulla. 2008. Nichtsprachliches als Textfaktor: Medialität, Materialität, Lokalität. Zeitschrift für Germanistische Linguistik 36(3). 343-354. 
Garcia, Angela C. \& Jennifer B. Jacobs. 1999. The eyes of the beholder: Understanding the turntaking system in quasi-synchronous computer-mediated communication. Research on Language and Social Interaction 32. 337-367.

Garfinkel, Harold. 1967. Studies in Ethnomethodology. Englewood Cliffs: Prentice-Hall. Gibson, James J. 1979. The Ecological Approach to Visual Perception. Boston: Houghton Mifflin.

Günthner, Susanne. 2011. Zur Dialogizität von SMS-Nachrichten - eine interaktionale Perspektive auf die SMS-Kommunikation. Networx 60.

Hausendorf, Heiko. 2003. Deixis and Speech Situation Revisited: The Mechanism of Perceived Perception. In Friedrich Lenz (ed.), Deictic conceptualisation of space, time and person (Pragmatics \& Beyond. New Series 112), 249-269. Amsterdam: John Benjamins.

Hausendorf, Heiko \& Wolfgang Kesselheim. 2008. Textlinguistik fürs Examen (Linguistik fürs Examen 5). Göttingen: Vandenhoeck \& Ruprecht.

Hausendorf, Heiko, Katrin Lindemann, Emanuel Ruoss \& Caroline Weinzinger. 2015. Ko-Konstruktionen in der Schrift? Zur Unterscheidung von Face-to-Face-Interaktion und Textkommunikation am Beispiel des Editierens fremder Beiträge in einem OnlineLernforum. In Ulrich Dausendschön-Gay, Elisabeth Gülich \& Ulrich Krafft (eds.), Ko Konstruktionen in der Interaktion: Die gemeinsame Arbeit an Äußerungen und anderen sozialen Ereignissen, 111-138. Bielefeld: transcript.

Hollway, Wendy. 1984. Gender Difference and the Production of Subjectivity. In Juan Henriques, Wendy Hollway, Cathy Urwin, Couze Venn \& Valerie Walkerdine (eds.), Changing the subject: Psychology, social regulation and subjectivity, 227-263. London: Methuen.

Huber, Ludwig. 2004. Forschendes Lernen: 10 Thesen zum Verhältnis von Forschung und Lehre aus der Perspektive des Studiums. die hochschule 2. 29-49.

Hutchby, Ian \& Vanita Tanna. 2008. Aspects of sequential organisation in text message exchange. Discourse and Communication 2(2). 143-164.

Kesselheim, Wolfgang \& Katrin Lindemann. 2010. Gemeinsam forschen lernen mit digitalen Medien: das Projekt „gi - Gesprächsanalyse interaktiv“. In Schewa Mandel, Manuel Rutishauser \& Eva Seiler Schiedt (eds.), Digitale Medien für Lehre und Forschung (Medien in der Wissenschaft 55), 106-117. Münster, New York: Waxmann.

Kesselheim, Wolfgang \& Katrin Lindemann. 2012. „gi - Gesprächsanalyse interaktiv“: Eine webbasierte Einführung in die Gesprächsanalyse auf der Grundlage kollaborativen forschenden Lernens. Gesprächsforschung Online Zeitschrift zur verbalen Interaktion 13. 118-142.

Kleinberger Günther, Ulla \& Franc Wagner. 2010. Wie schreiben Schülerinnen und Schüler in neuen Medien? In Eva-Maria Jakobs, Katrin Lehnen \& Kirsten Schindler (eds.), Schreiben und Medien: Schule, Hochschule, Beruf (Textproduktion und Medium 10), 37-50. Frankfurt a.M., Bern, New York: Lang.

Lehnen, Katrin \& Eva-Maria Jakobs. 2003. Writing Well Online: Talent Isn’t Enough. Netzspezifische Schreibkompetenz. In Konrad Ehlich \& Angelika Streets (eds.), Wissenschaftlich schreiben lehren und lernen, 391-407. Berlin: de Gruyter.

Lindemann, Katrin, Emanuel Ruoss \& Caroline Weinzinger (i. Dr.): Lesen und gelesen werden. „Lesepräsenz“ in schriftlicher Kommunikation. In Jianhua Zhu, Jin Zhao \& Michael Szurawitzki (eds.): Akten des XIII. Germanistenkongresses Shanghai 2015 „Germanistik zwischen Tradition und Innovation“. Band 3 Frankfurt a.M., Bern, New York: Lang.

Lucius-Hoene, Gabriele \& Arnulf Deppermann. 2004. Narrative Identität und Positionierung. Gesprächsforschung Online Zeitschrift zur verbalen Interaktion 5. 166-183. 
Luckmann, Thomas. 1988. Grundlagen der Soziologie: Strukturen sozialen Handelns: Kurseinheit 3. Hagen: FernUniversität Hagen: Fachbereich Kultur- und Sozialwissenschaften.

Luhmann, Niklas. 1984. Soziale Systeme: Grundriss einer allgemeinen Theorie. Frankfurt a.M.: Suhrkamp.

Portmann-Tselikas, Paul R. 2005: Was ist Textkompetenz? http://tiny.uzh.ch/y6 «01.05.2016〉.

Psathas, George (ed.) 1990. Interaction competence (Studies in ethnomethodology and conversation analysis 1). Washington DC: International Institute for Ethnomethodology and Conversation Analysis; University Press of America.

Reiber, Karin. 2007. Forschendes Lernen als Leitprinzip zeitgemäßer Hochschulbildung. In Karin Reiber (ed.), Forschendes Lernen als hochschuldidaktisches Prinzip Grundlegung und Beispiele (Tübinger Beiträge zur Hochschuldidaktik 3/1), 6-12. Tübingen.

Roth, Kersten S. \& Jürgen Spitzmüller (eds.) 2007. Textdesign und Textwirkung in der massenmedialen Kommunikation (Kommunikationswissenschaft). Konstanz: UVK.

Sacks, Harvey. 1992. Lectures on Conversation. Volumes I \& II. Ed. by Gail Jefferson; with an introduction by Emanuel A. Schegloff. Oxford, Cambridge: Blackwell.

Sacks, Harvey \& Emanuel A. Schegloff. 1979. Two preferences in the organization of reference to persons in conversation and their interaction. In George Psathas (ed.), Everyday Language: Studies in Ethnomethodology, 15-21. New York: Irvington Press.

Sacks, Harvey, Emanuel A. Schegloff \& Gail Jefferson. 1974. A Simplest Systematics for the Organization of Turn-Taking for Conversation. Language 50(4). 696-735.

Schegloff, Emanuel A. 1968. Sequencing in Conversational Openings. American Anthropologist 70(6). 1075-1095.

Schegloff, Emanuel A. 1972. Notes on a conversational practice: Formulating place. Studies in social interaction. 75-119.

Schegloff, Emanuel A. 1996. Some Practices for Referring to Persons in Talk-in-Interaction: A Partial Sketch of a Systematics. In Barbara A. Fox (ed.), Studies in Anaphora (Typological studies in language 33), 437-485. Amsterdam: Benjamins.

Schegloff, Emanuel A. \& Harvey Sacks. 1973. Opening up Closings. Semiotica 8. 289-327.

Spiegel, Carmen \& Ulla Kleinberger Günther. 2006. Schreiben im Internet als neue Aufgabe der Didaktik. In Carmen Spiegel \& Rüdiger Vogt (eds.), Vom Nutzen der Textlinguistik für den Unterricht, 187-199. Baltmannsweiler: Schneider Verlag Hohengehren.

Steinseifer, Martin. 2010. Textroutinen im wissenschaftlichen Schreiben Studierender: Eine computerbasierte Lernumgebung als Forschungs- und Lerninstrument. In Eva-Maria Jakobs, Katrin Lehnen \& Kirsten Schindler (eds.), Schreiben und Medien: Schule, Hochschule, Beruf (Textproduktion und Medium 10), 93-114. Frankfurt a.M., Bern, New York: Lang.

Wolff, Stephan. 2006. Textanalyse. In Ruth Ayaß \& Jörg R. Bergmann (eds.), Qualitative Methoden der Medienforschung (rororo 55665), 245-273. Reinbek: Rowohlt. 